\title{
An Approach for Enriching Information for Supporting Collaborative e-Work
}

\author{
Obinna Anya, Atulya Nagar, and Hissam Tawfik \\ Intelligent and Distributed Systems Lab, Deanery of Business and Computer Sciences, \\ Liverpool Hope University, Liverpool, United Kingdom L16 9JD \\ $\{05008721$, nagara, tawfikh\} @hope.ac.uk
}

\begin{abstract}
As a result of the high level of knowledge required in collaborative eWork as well as its changing work contexts, e-Work support systems need to provide not only information in the form of documents and articles, but also expert-level explanations in the form of supporting literature and references to theories and related cases, to justify retrieved information and offer cognitive support to e-Work. In this paper, we present a novel approach for enriching information for supporting collaborative e-Work, which combines latent semantic analysis, domain task modelling and conceptual learning. We illustrate the potential of our approach using our e-Workbench system. e-Workbench is a prototype system for adaptive collaborative e-Work.
\end{abstract}

Keywords: Collaborative e-Work, information enrichment, concept-based knowledge acquisition, cognitive support.

\section{Introduction}

Collaborative e-Work is highly knowledge-intensive, and involves experts, often, with different knowledge backgrounds and from different work organisations who share knowledge in order to arrive at an optimal decision or problem-solving strategy [2], [9]. Such work activity requires both documented and experience-based knowledge. As a result, collaborative e-Work support systems need to provide not only information in the form of documents and articles, but also expert-level explanations in the form of supporting literature and references to theories and related cases to justify retrieved information and offer cognitive support to augment workers' ideas.

Different techniques have been proposed in advanced information processing, the semantic Web and knowledge management, towards information enrichment. In machine learning, natural language processing and information retrieval, various techniques have been combined in efforts to identify features for indexing information resources. A number of these methods employ shallow statistical inferences that do not typically result in knowledge-rich representations. Techniques in natural language processing have been explored but their huge reliance on grammar makes them less attractive, especially in domains where problem-solving methodologies have not recorded using strict grammatical structure [3]. Within the Semantic Web community, the use of metadata annotations, such as tagging [4] has proved immensely popular as a technique for enriching information resources, but its employment of domain experts makes it less cost-effective. 
This paper presents a novel approach for enriching information for supporting collaborative e-Work, which combines latent semantic analysis (LSA), domain task modelling (DTM) and conceptual learning. The focus is to enable the work environment to acquire sufficient knowledge of a work domain in terms of key terms and concepts within the domain and their relationships, (previous) cases, as well as possible tasks and task goals in order to provide expert-level explanations to justify retrieved information resources and offer cognitive support to augment workers' ideas. We illustrate the potential of our approach using e-Workbench [5].

\section{Related Work}

A number of researches have been carried in areas, such as advanced information processing, computer supported cooperative work and group decision making, knowledge management and the semantic Web, towards information enrichment for various purposes. Feng et al. [1] proposed a model of an information space, consisting of knowledge and document subspaces, to enable the acquisition of knowledge from digital libraries. The authors noted that two shortcomings in the effort towards satisfying man's information needs to support decision making are inadequate strategic level cognition support and inadequate knowledge sharing facilities. Ackerman [8] presented the Answer Garden, which is aimed to achieve knowledge acquisition in two intertwined ways - by making relevant information retrievable and by making people with knowledge accessible. The issue of common understanding in collaborative decision making is addressed in [7] through argumentative discourse and collaboration. Evangelou et al. [6] presented an approach for supporting knowledge-based collaborative decision making, which aims at developing knowledge management services for the capturing of organisational knowledge in order to augment teamwork, and thus enhance decision making quality.

An underlying goal of most of these efforts is to analyse information and/or integrate multiple knowledge resources so as to derive a semantic relationship among the basic concepts. The novelty in our approach lies in the ability to employ an integrated approach aimed to enable a work support system to acquire sufficient knowledge of a work domain in order to cognitively provide expert-level explanations to justify the use of an information resource.

\section{3 e-Workbench Overview}

We developed e-Workbench in [5], a prototype intelligent system aimed to equip future collaborative workspaces to adapt to work, and creatively support problem solving and decision making by leveraging on distributed knowledge resources in order to proactively augment workers' capabilities. The ultimate goal is to enable the work environment to become not only a workspace, but also 'a co-worker' and 'a collaborator' as a result of its knowledge of work and creative participation in problem solving and decision making.

We use a semi-automated approach in enabling e-Workbench to acquire knowledge of work and appropriately understand the users' domain of work. This involves: 
(1) 'Training' e-Workbench to learn about key concepts and threads of ideal problem solving strategies within the domain of work. (2) Generating knowledge element models (KEMs) of the domain based on a DTM as well as cases and tasks that constitute best problem solving strategies. With the knowledge acquired during training and the KEMs generated, e-Workbench is able to retrieve appropriate information to assist in decision making and provide justifications for e-workers' views. Fig. 1 depicts an overview of the e-Workbench approach.

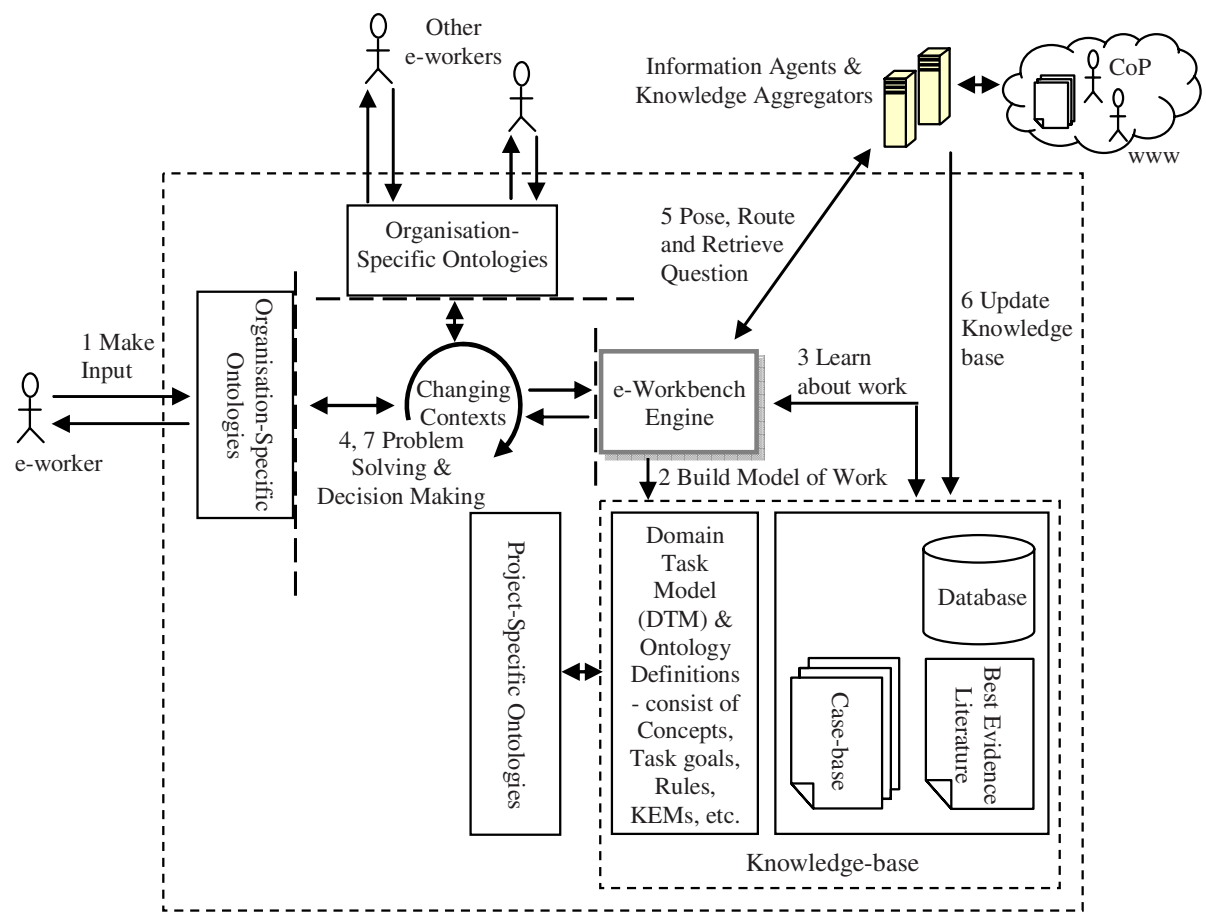

Fig. 1. The e-Workbench Approach

\section{Information Enrichment Approach}

Our approach to information enrichment for supporting collaborative e-Work involves the following processes:

- Posing a question to a knowledge expert or an information retrieval agent such as a search engine, and obtaining retrieved results.

- Selecting the most appropriate result(s) based on domain expert knowledge.

- Using LSA to infer deeper meanings from the selected documents.

- Using DTM, conceptual learning and project-specific ontologies to construct concept structures, relevant cases and KEMs based on work context tree (WCT) in order to acquire contextual knowledge about work. 
- Mapping the knowledge extracted to the meanings inferred from the selected documents in order to conceptually enrich retrieved information.

During collaborative problem solving and decision making, information is usually retrieved when a user poses a question to a knowledge expert or a search engine in order to resolve an exception. Our experience in developing e-Workbench, however, shows that this process of enriching the retrieved information cannot be completely automated particularly with regard to e-Work because of its rapidly changing work contexts and the likely use of loosely coupled teams from multiple domains of expertise. As a result, we use a semi-automatic approach, which is described as follows, to achieve this.

\subsection{Query Analysis}

A search query is manually elaborated by a domain expert in order to accentuate relevant terms and domain concepts that relate to the query. The elaboration process involves (1) extracting representative terms, which are likely to contain domain knowledge and which actually contain the expected results from a given query, and (2) incorporating relevant terms that may not appear in the query, but could be used to capture key concepts and domain rules within the domain. The extracted and incorporated terms are used to build an accentuated matrix of key terms/concepts used during the LSA-based indexing process (see sec. 4.4). During the elaboration process, terms with different names across different sub-domains, e.g. civil engineering, building site and estate development, which semantically refer to the same object, are identified. We refer to such terms as synonyms. On the other hand, terms, which we refer to as polysemys that have different shades of meaning across sub-domains, are equally identified. Our elaboration approach enables us to solve the problem of the apparent inability of Web users to formulate effective search queries that accurately reflect their information needs [10], which thus poses the challenge of finding the optimum search word size of a query that will give the most effective search result. Our technique is to identify the key terms within a search query, or that are related to a search query, their synonyms and polysemys, accentuate those terms within the query, and relate them to the concept and context of work for effective knowledge support. The accentuated terms from the search query may also be used as subsequent queries. Based on our conceptual query analysis, each term is related to a domain concept, and relevant documents selected from the retrieved query results.

\subsection{Conceptual Learning}

Using a conceptual learning mechanism [11], e-Workbench is trained on the domain of work. The goal of conceptual learning is to enable the system to build a "knowledge space" for understanding relevant concepts, principles and facts within the identified domain(s), and a "conceptual space" for inferring relationships among them. This forms the semantic memory [12] of e-Workbench, which allows it to build the 
cognitive capabilities required for accurate perception of concepts (and objects) within the domain of work. In intelligent systems, perception is accomplished by the ability of a system to recognise and analyse features of concepts (and objects), which it encounters (and interacts with). For a given search query, we identify two levels of concepts: the high level composite concept and the low level primitive concept. Using simple mapping rules, we denote these generically, and using an example from the domain of civil construction, as:

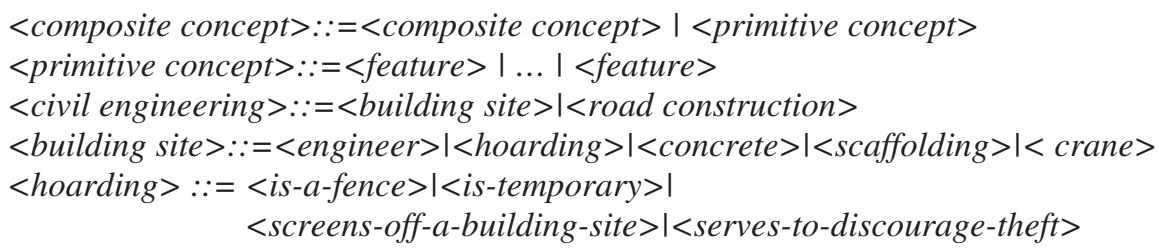

A feature is a psychological representation of properties of the world that can be processed independently of other properties and that are relevant to a task, such as categorisation, in collaborative e-Work. They are identified by their functional role in cognitive processing, such as the act of including (or excluding) an entity as a member of a category [13]. We represent a feature as a 2-tuple:

feature $(V, T)$

where $\mathrm{V}$ is a set of properties of a feature and $\mathrm{T}$, a set of types for corresponding properties. $\mathrm{V} \neq \phi ; \mathrm{T} \neq \phi$.

The next stage of our conceptual learning technique consists of building the episodic memory [12] of e-Workbench. Episodic memory is used for storing events (within collaborative e-Work) having features of a particular time and space, and for storing episodes and traces of learning - about skills, problem solving strategies and processes, decision making procedures, contextual use of information resources - that occur within relevant cases through case-based reasoning. Episodic memory is associative in nature and content-addressable [12]. As a result, it could be used to appropriately hold episodic information about a case in collaborative e-work by storing tasks based on concept as well as context, storing logical associations between tasks, specifying appropriate action plans and storing dynamic references to information resources. In e-Workbench, this memory is populated with cases, which are, in turn, composed of conceptually categorised tasks. A case and a task are both represented as a 3-tuple entity as follows:

case $(P, A, S)$

task $(C, R, E)$

where $\mathrm{P}$ is the problem (or case) description, which includes the initial problem state, relevant concepts and their feature values, a description of problem execution context and possible actors (people and virtual roles), one or more tasks and their (sub)goals as well as associated action plans or behaviour streams required to achieve those goals; A is an action plan or behaviour stream whose execution transforms the problem from the problem state to a goal state; and $\mathrm{S}$ is the expected result when $\mathrm{A}$ is 
applied to P. C is the case under which the task is valid or, at least, applicable, R is the set of resources required to perform the task, and $\mathrm{E}$ is the set of operations of corresponding resources.

\subsection{Domain Task Modelling}

The domain concepts related to the given search query are manually analysed using WCT [5]. The goal is to filter out, from the DTM, possible motives of a worker's actions and ideas, and correlate them conceptually based work goal. To achieve this, we analyse the given e-Work project in terms of the domain(s), existing knowledge, given terms of reference, previous cases and possible tasks and task goals. The work context tree enables us to generate semantically rich service descriptions used to meaningfully encapsulate ideas and knowledge resources within an e-Work task structure. We refer to these semantic rich service descriptions as KEMs. We use a KEM to refer to concept knowledge, which could be described by an information resource (i.e., an entity that has identity, for example, given by a URI) that is capable of supplying coherent statements of facts, empirical results, reasoned decisions or ideas (i.e., data, information, processes), which can be applied to justify the use of retrieved information in collaborative problem solving and decision making, to corroborate or refute a worker's view or to build new knowledge.

Fig. 2 shows a WCT. The root, KW, of the top-down tree is the given e-Work project or problem of interest. The root node contains three items: the domain ontology, $\mathrm{D}$, which provides domain permissible procedures, rules and conceptual information relevant to KW, existing knowledge, $\mathrm{K}$ and work goal, G. K comprises theories, stories, hypotheses, philosophies, assertions, rules, metaphors and initial work input, in the form of terms of references, relevant to KW. G is the expected result of work. The next level consists of a set of nodes that describes cases within the KW. Each case node contains two items: the work, $\mathrm{KW}$ (as defined in the root node) and the case context, $\mathrm{C}_{\mathrm{C}} \cdot \mathrm{C}_{\mathrm{C}}$ comprises goals, motives, conditions and information that pertain to the case. The third level consists of nodes that describe tasks in the KW. Each task node consists of three items: the next upper level case node, $\mathrm{C}_{\mathrm{x}}(1 \leq x \leq n)$, the task context, $\mathrm{C}_{\mathrm{T}}$ and the task goal, $\mathrm{O}$. The fourth level (the leaves) consists of the KEMs. A KEM has four items: the next upper level task, $\mathrm{T}_{\mathrm{x}}(1 \leq x \leq n)$, a knowledge descriptor, $\mathrm{S}$, the role, $\mathrm{R}$, the effect, $\mathrm{E}$ and $\mathrm{N}$, the referred cognitive node(s). The knowledge descriptor provides metadata descriptions about the KEM. $\mathrm{R}$ is the action performed or knowledge supplied by KEM, while $\mathrm{E}$ is the expected change brought about by $\mathrm{R}$ in $\mathrm{T}_{\mathrm{x}}$. $\mathrm{N}$ refers to the node(s) in a knowledge network that possibly has (or have) the resource (information, service or human expertise) required to perform $T_{\mathrm{x}}$, or augment the process of performing $T_{x}$ or taking a decision towards performing $T_{x}$. $\mathrm{N}$ could be Web resources, denoted by a URL, non-Web resources, e.g. a book, human agents, such as an expert in the given domain of work or non-human agents, such as knowledge repositories, Web sites, content and referral databases, avatars, and "webbots" [14] that have additional information with which to support retrieved information. The three nodes provide three cognitive planes, with which to analyse work at the domain, conceptual and task levels. 


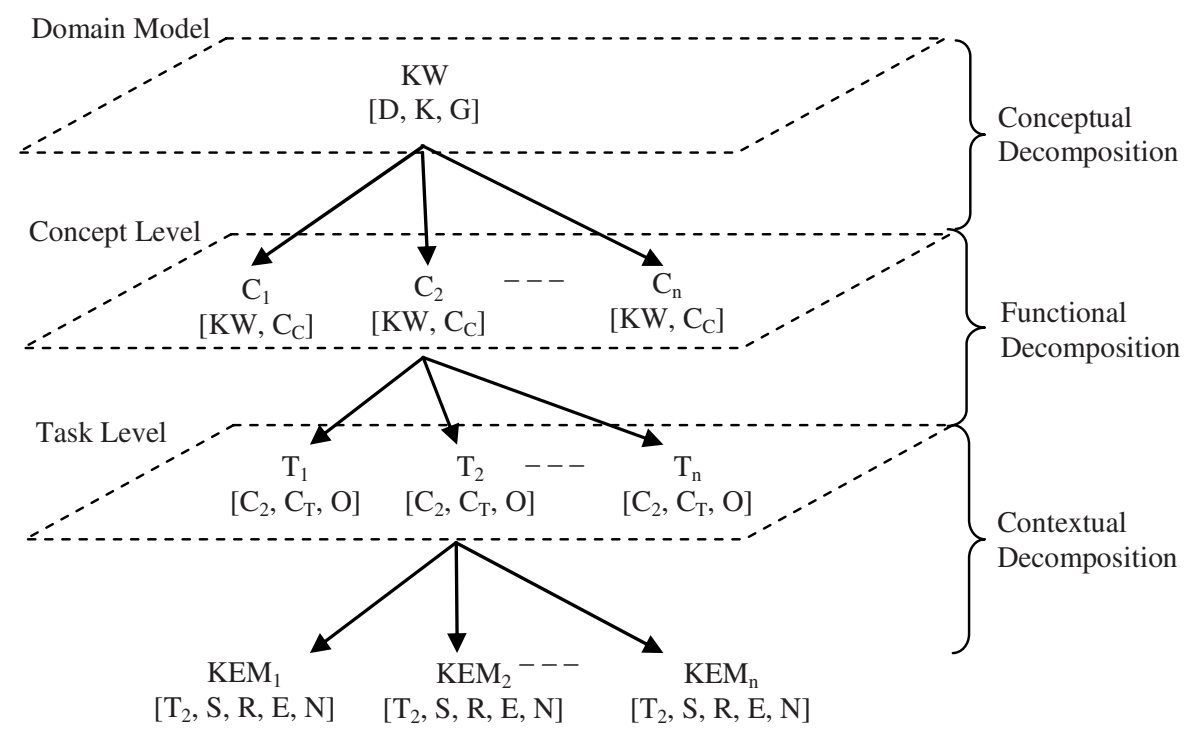

Fig. 2. Work Context Tree for Generating Knowledge Element Models

\subsection{Latent Semantic Analysis}

We use LSA [15] to infer deeper meanings and relations from the words, phrases and sentences in an elaborated search query, and associate those meanings to the documents retrieved. Using LSA, we aim to enable the system to predict what terms "actually" are implied by a query or apply to a retrieved document. Table 1 shows four documents D1, D2, D3 and D4, which are the selected results from the query: importance of hoarding materials. Key terms from the elaborated search query are shown on the first row of the table. The key concept being considered is building construction, and is represented in the table by p. The context of work, q, is described by the phrase "a building construction site".

From the table, the user would have considered documents 2, 3 and 4 relevant (column R). Document 2 contains words within the context of work, while document 3 contains a key word, but relates to neither the underlying concept nor the context of work. Document 1, though not considered relevant by the user, matches the query because it relates to the underlying concept, while document 4 matches the query because it relates to both the concept and context of work.

Table 1. Sample Term by Document Matrix

\begin{tabular}{|c|c|c|c|c|c|c|c|c|c|}
\hline & hoarding & building & material & board & fence & $\mathrm{p}$ & $\mathrm{q}$ & $\mathrm{R}$ & $\mathrm{M}$ \\
\hline D1 & $*$ & $*$ & & & & $*$ & & & $*$ \\
\hline D2 & & & $*$ & $*$ & $*$ & & $*$ & $*$ & \\
\hline D3 & $*$ & & & & & & & $*$ & \\
\hline D4 & $*$ & $*$ & $*$ & & & $*$ & $*$ & $*$ & $*$ \\
\hline
\end{tabular}


LSA uses the technique of singular value decomposition in which a rectangular matrix, A of terms by documents, $\mathrm{m} \mathrm{x} \mathrm{n}$, is decomposed into a product of three other matrices from which the original matrix can be approximated by linear combination, as denoted by eqn. 1 :

$$
\mathrm{A}_{(\mathrm{mxn})}=\mathrm{T}_{\mathrm{O}(\mathrm{mxm})} \mathrm{S}_{\mathrm{O}(\mathrm{mxn})} \mathrm{D}_{\mathrm{O}(\mathrm{nxn})^{\prime}} .
$$

such that $\mathrm{T}_{\mathrm{O}}$ represents the term matrix, $\mathrm{D}_{\mathrm{O}}$ ' represents the document matrix, and $\mathrm{S}_{\mathrm{O}}$, a diagonal matrix containing singular values arranged in descending order. Our goal is to capture the significance of every term in the query to the underlying concept of work. As shown in fig. 3, the g highest singular values identify the g most importance concepts in $T_{O}$, which is represented by a $T_{(m x g)}$ matrix. The weights in $S_{(g x g)}$ reflect the importance of concepts in $\mathrm{T}_{(\mathrm{mxg})}$. Multiplying $\mathrm{T}_{(\mathrm{mxg})}$ by $\mathrm{S}_{(\mathrm{gxg})}$ results in the accentuation of the entries (concepts) in $\mathrm{T}_{(\mathrm{mxg})}$.
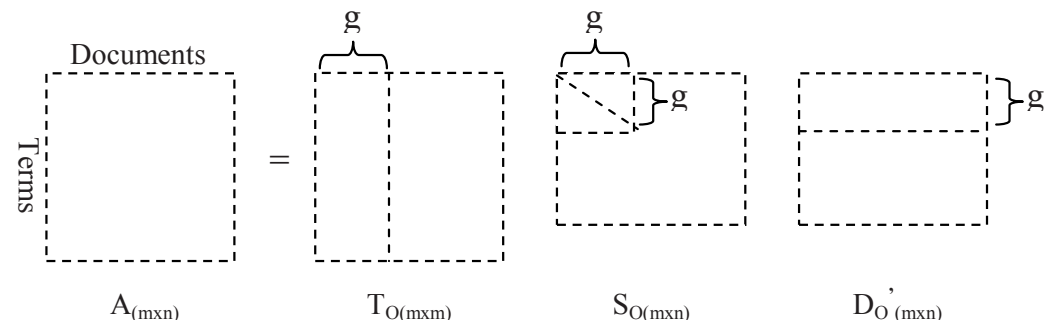

Fig. 3. Singular Value Decomposition of a Term by Document Matrix

\subsection{Enriching Retrieved Information}

Fig. 4 shows our model for mapping extracted concepts to acquired knowledge in order to enrich retrieved information for supporting collaborative e-Work. The model consists of a knowledge space (KS), an information space (IS) and a cognition support area (CS). A retrieved information resource is analysed (see sec. 4.1) and input into the knowledge space, and KEMs are generated from it based on fig. 2. KS contains the DTM for the given e-Work project, ontology definitions and work goal as well as concept models, cases and possible tasks and task goals. In e-Workbench, ontologies are defined based on the combined ontologies and knowledge structure of all organisations participating in the given e-Work project as well as the DTM of the e-Work project (see fig. 1). Within the IS, users and agents search for relevant documents using the generated KEMs and key terms as search guide. From the generated KEMs, a KEM is selected. Work context information is applied to it; and best evidence literature information, used to enrich it to provide justifications for retrieved resources. Concepts and deeper meanings are extracted from the retrieved documents (see sec. 4.2 - sec. 4.4) to provide explanations and enhance their information base. Finally cognition support (expert-level knowledge) is provided in the CS in the form of rich information (i.e. enhanced retrieved document + supporting explanations and justifications. 


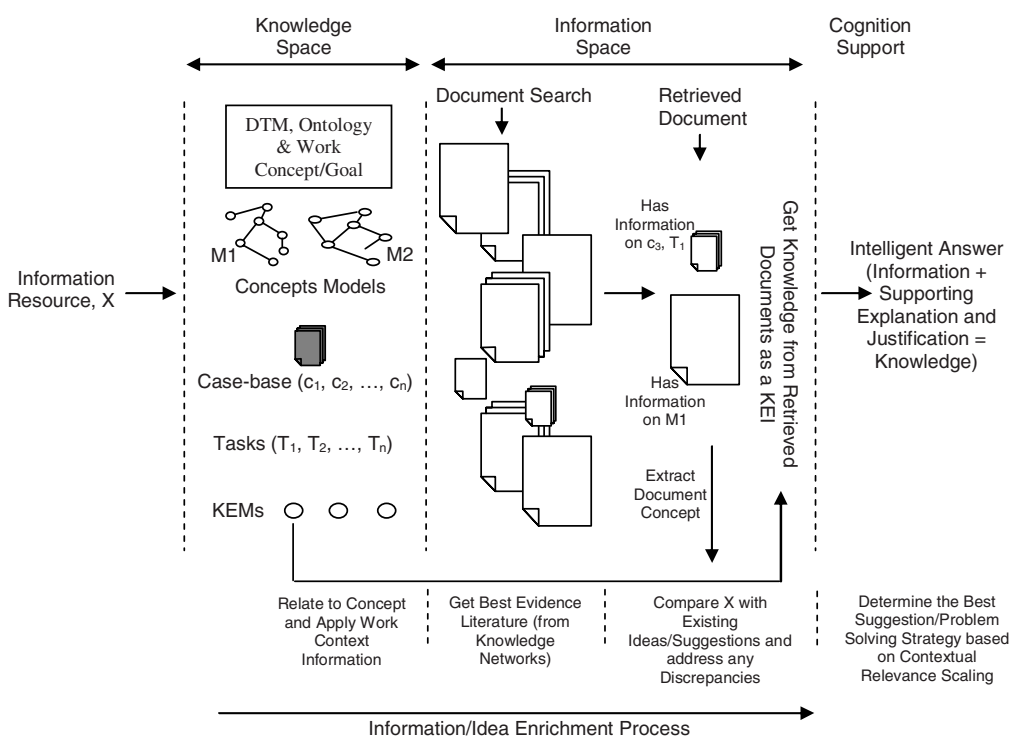

Fig. 4. Information Enrichment Framework

\section{Conclusion and Future Work}

This paper presents an approach for enriching information for supporting collaborative e-Work, which combines LSA, DTM and conceptual learning. The focus is to enable the work environment to acquire knowledge of the domain of work in terms of key terms and concepts within the domain and their relationships to previous cases, as well as possible tasks and task goals in order to provide expert-level explanations to justify retrieved information resources, and offer cognitive support to augment workers' ideas. Our future work will focus on developing mechanisms to keep track of the changing work contexts in collaborative e-Work so as to ensure that enriched information resources are effectively used to cognitively support decision making and problem solving.

\section{References}

1. Feng, L., Jeusfeld, M., Hoppenbrouwers, J.: Beyond Information Searching and Browsing: Acquiring Knowledge from Digital Libraries. INFOLAB Technical Report ITRS-008, Tilburg University, The Netherands (February 2001)

2. Nof, S.: Design of Effective e-Work: Review of Models, Tools, and Emerging Challenges. Production Planning and Control 14(8), 681-703 (2003)

3. Asiimwe, S., Craw, S., Wiratunga, Taylor, B.: Automatically Acquiring Structured Case Representations: Smart Way. In: Ellis et al. (eds.) Applications \& Innovations in Intelligent Systems XV, pp. 45-58. Springer, London (2007) 
4. Tanasescu, V., Streibel, O.: Extreme Tagging: Emergent Semantics through the Tagging of Tags. In: Proc. of the Int.l Workshop on Emergent Semantics \& Ontology Evolution, ISWC/ASWC 2007, Busan, S Korea (2007)

5. Anya, O., Nagar, A., Tawfik, H.: A Conceptual Design of an Adaptive and Collaborative e-Work Environment. In: Proc. of the 1st Asian Modelling Symposium, Asia 2007, Thailand, March 27-30 (2007)

6. Evangelou, C., Karacapilidis, N., Tzagarakis, M.: On the Development of Knowledge Management Services for Collaborative Decision Making. Journal of Computers 1(6), 19 28 (2006)

7. Karacapilidis, N., Papadias, D.: Computer-Supported Argumentation and Collaborative Decision Making: The Hermes System. Information Systems 26(4), 259-277(19) (2001)

8. Ackerman, M.: Augmenting the Organizational Memory: A Field Study of Answer Garden. In: Proc of the ACM Conf on Computer Supported Cooperative Work (CSCW 1994), October 22-26,1994, pp. 243-252. ACM, Chapel Hill, NC (1994)

9. Experts Group: New Collaborative Working Environments 2020, EUROPEAN COMMISSION Information Society Directorate-General, Report of the Experts Group on Collaboration@Work, Brussels (February 2006)

10. Smyth, B.: Adventure in Personalised Web Search. In: proc of 27th SGAI Int'l Conf on Innovative Techniques and Applications of AI, Cambridge, December 12-14 (2007)

11. Wiig, E., Wiig, K.: On Conceptual Learning, Knowledge Research Institute, Inc. Working Paper 1999-1 (1999)

12. Ramamurthy, U., D'Mello, S., Franklin, S.: Modified Sparse Distributed Memory as Transient Episodic Memory for Cognitive Software Agents. In: IEEE Int'l Conf on Systems, Man and Cybernetics, vol. 6, pp. 5858-5863 (October 2004)

13. Rogosky, B., Goldstone, R.: Adaptation of Perceptual and Semantic Features. In: Carlson, L., van der Zee (eds.) Functional Features in Language and space: Insights from perception, categorisation and development, pp. 257-273. Oxford University Press, Oxford (2005)

14. Carley, K.: Smart agents and organizations of the future. In: Lievrouw, L., Livingstone, S. (eds.) Handbook of new media, pp. 206-220. Sage, London (2002)

15. Deerwester, S., Dumais, S., Furnas, G., Landauer, T., Harshman, R.: Indexing by latent semantic analysis. Journal of the American Society for Information Science 41(6), 391407 (1990) 